\title{
Aggressive radiolucent lesion of the mandible $\$$
}

Vitor Bonetti Valente, DDS, ${ }^{\mathrm{a}}$ Icléia Siqueira Barreto, MD, MS, PhD,${ }^{\mathrm{b}}$ Cristiane Furuse, DDS, MS, PhD, ${ }^{\mathrm{c}}$

Éder Ricardo Biasoli, DDS, MS, PhD, ${ }^{\mathrm{d}}$ Glauco Issamu Miyahara, DDS, MS, PhD, ${ }^{\mathrm{d}}$ and

Daniel Galera Bernabé, DDS, MS, $\mathrm{PhD}^{\mathrm{e}}$

(Oral Surg Oral Med Oral Pathol Oral Radiol 2016;122:265-271)

\section{CLINICAL PRESENTATION}

The patient, a 76-year-old white woman, was seen at the Oral Oncology Center, Araçatuba Dental School, São Paulo State University - UNESP, São Paulo, Brazil, for evaluation of a lesion located in the body and angle of the mandible. The patient reported pain and swelling that had started about 2 months previously. Her medical history revealed diabetes and hypertension, as well as treatment for adenocarcinoma of the colon 20 years ago. In addition, the patient reported that she was an ex-smoker, having stopped 22 years ago. Extraoral clinical examination revealed a swelling of fibrous consistency in the body and angle on the right side of the mandible (Figure 1A). During intraoral examination, a non-ulcerated swelling involving the right side of the mandible was also observed (Figure 1B). No submandibular and cervical lymphadenopathy was detected. Radiographic examination, computed tomography (CT), and three-dimensional (3-D) reconstruction showed an expansive and destructive bone lesion in the body and angle of the mandible, extending to the ramus (Figure 2). Significant disruption of the vestibular and lingual cortices was also detected.

\section{DIFFERENTIAL DIAGNOSIS}

A wide range of diagnosis can be generated for lesions causing bone destruction in the body and angle of the

This case was reported in the XXII Brazilian Congress of Oral Medicine and Oral Pathology on July 2014 in the Sociedade Brasileira de Estomatologia e Patologia Oral meeting.

${ }^{\mathrm{a}}$ MS Student in Stomatology, Oral Oncology Center, Department of Pathology and Clinical Propedeutics, Araçatuba Dental School, UNESP - Universidade Estadual Paulista, Araçatuba, São Paulo, Brazil.

${ }^{b}$ Medical Assistant, Department of Pathology, School of Medicine, University of Campinas, UNICAMP, Campinas, São Paulo, Brazil.

${ }^{\mathrm{c} A s s i s t a n t}$ Professor, Department of Pathology and Clinical Propedeutics, Araçatuba Dental School, UNESP - Universidade Estadual Paulista, Araçatuba, São Paulo, Brazil.

${ }^{\mathrm{d}}$ Adjunct Professor, Oral Oncology Center, Department of Pathology and Clinical Propedeutics, Araçatuba Dental School, UNESP Universidade Estadual Paulista, Araçatuba, São Paulo, Brazil.

${ }^{\mathrm{e}}$ Assistant Professor, Oral Oncology Center, Department of Pathology and Clinical Propedeutics, Araçatuba Dental School, UNESP Universidade Estadual Paulista, Araçatuba, São Paulo, Brazil.

Received for publication Mar 16, 2015; returned for revision Nov 2, 2015; accepted for publication Nov 9, 2015.

(c) 2016 Elsevier Inc. All rights reserved.

2212-4403/\$ - see front matter

http://dx.doi.org/10.1016/j.0ooo.2015.11.002 mandible, associated with extraoral swelling. However, before microscopic analysis, some clinical and imaging aspects may guide the clinician to make a checklist of the most probable diagnostic hypotheses. Thus, based on previous medical history, clinical and radiographic examination, and findings from maxillofacial CT and 3$\mathrm{D}$ reconstruction of the present case, our main diagnostic hypotheses included an odontogenic tumor (OT) with aggressive behavior, metastatic adenocarcinoma of the colon (MAC), osteosarcoma of the jaws (OSJ), chondrosarcoma, malignant odontogenic lesions, and other rare sarcomas.

OTs are uncommon lesions of the mandible and maxilla, constituting $0.7 \%$ to $2.5 \%$ of all oral biopsy specimens, depending on the study and country. ${ }^{1}$ However, a number of benign and malignant OTs should be considered in the main differential diagnosis for destructive bone lesions of the mandibular body and angle. Most conventional ameloblastomas (80\%$85 \%$ ), for example, occur in the posterior mandible (molar-ramus area). ${ }^{2}$ These benign entities appear with equal frequency in both genders and also with equal prevalence in the third to seventh decades of life. ${ }^{2}$ OTs, especially ameloblastomas, may be detected incidentally on radiologic imaging, ${ }^{3}$ but in a few patients, signs and/or symptoms, such as a slowgrowing, painful swelling, are present, ${ }^{1,2}$ as we observed in this case. However, ameloblastomas can reach a very large size, causing facial disfigurement and functional impairment. ${ }^{2}$ Keratocystic odontogenic tumor (KOT) was also considered in the present case. KOT can be found in patients in a wide age range (about $60 \%$ of cases are diagnosed between 10 and 40 years) in both genders. ${ }^{2}$ In general, this tumor occurs in the mandible (60\%-80\%). ${ }^{2}$ Radiographically, KOT shows a radiolucent area with well-defined sclerotic margins, ${ }^{2}$ but this feature was not consistent with our case. Likewise, it would be unusual for a KOT to break through the cortex and to cause significant pain. Myxoma is another aggressive OT that may be included in the differential diagnosis of a large destructive lesion in the posterior mandible. ${ }^{1,2}$ This tumor is commonly found in young adults (mean age 2530 years) with similar gender distribution; normally, it can be observed as a radiolucent lesion with irregular margins. ${ }^{2}$ In the radiographic examination, CT, and 3-D reconstruction, the extensive bone destruction in our 

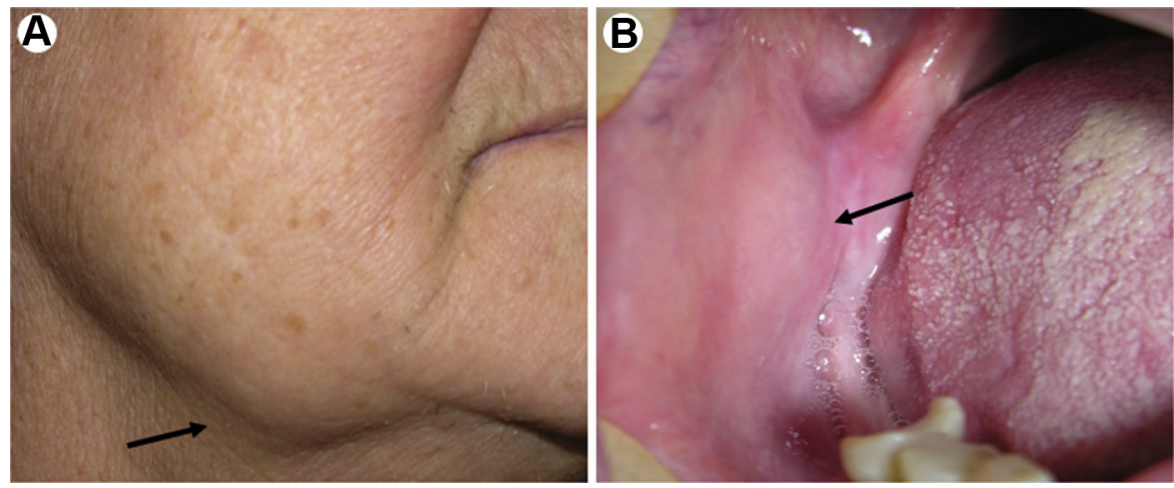

Fig. 1. Facial (A) and intraoral (B) non-ulcerated swelling involving the right side of the mandible (arrows). The lesion caused a painful, slow-growing vestibular and lingual mass in the region of body and angle, affecting the local mucosa and adjacent tissues.
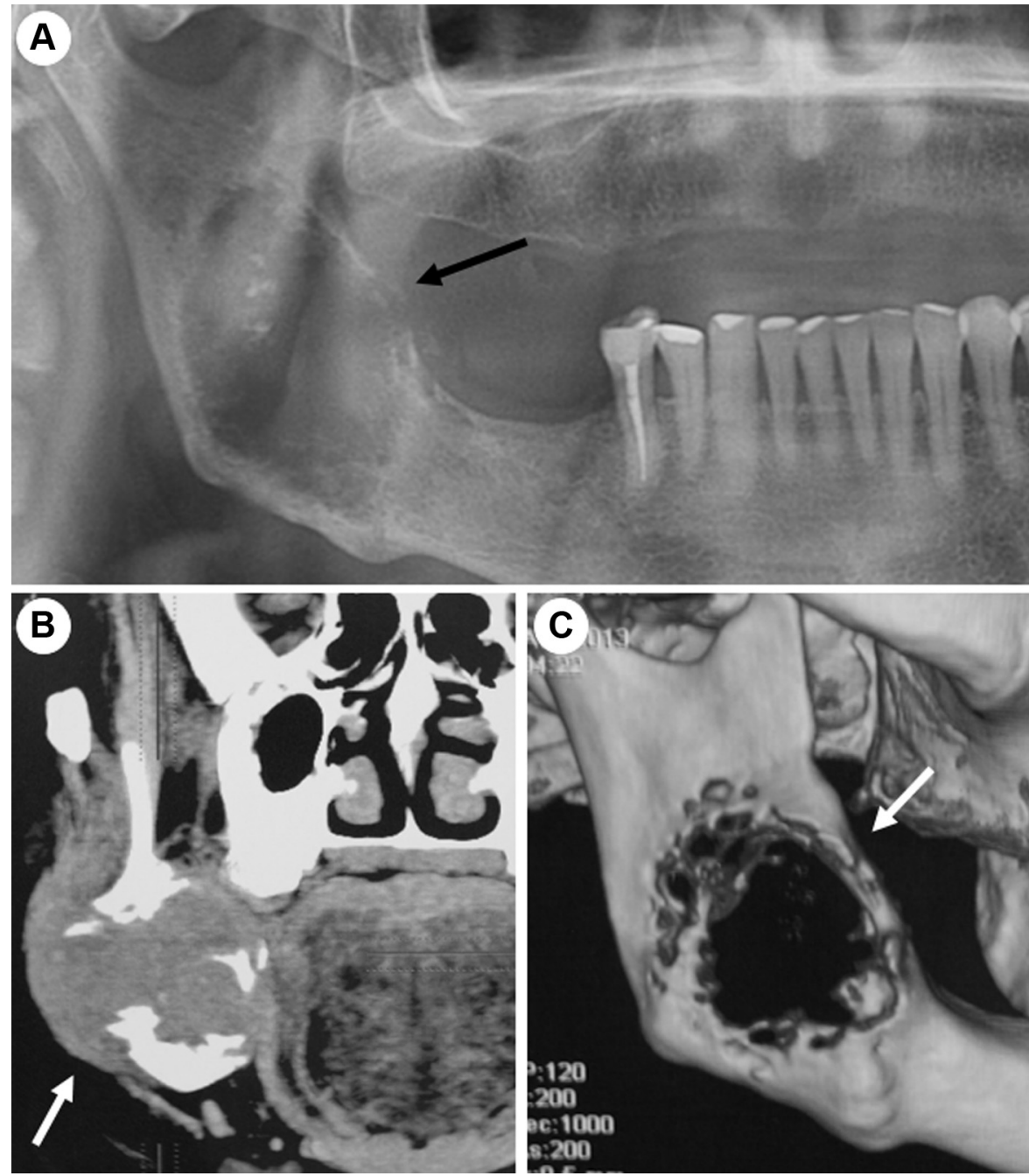

Fig. 2. Radiographic examination (A); computed tomography in a coronal section (B); and three-dimensional reconstruction (C) showing a destructive and expansive bone lesion located in the body, angle, and ramus of the mandible on the right side (arrows).

patient could be observed clearly, as would be found in cases of an aggressive OT.

Nonodontogenic jaw lesions, such as central giant cell granuloma and aneurysmal bone cyst, might be included in the differential diagnosis for this case, although the patient's age makes these lesions unlikely. The central giant cell granuloma can be found before 30 years of age in more than $60 \%$ of all cases, with 
approximately $70 \%$ in the mandible and most of the lesions appearing in the anterior region, crossing the middle line. ${ }^{2}$ Aneurysmal bone cysts in the jaws are uncommon lesions (about 2\% of all cases reported) ${ }^{2}$ and typically affect patients in the first 2 decades of life. ${ }^{4}$ The vast majority of cases are found in the posterior segments of the mandible. ${ }^{2}$

Metastatic carcinomas involving the oral cavity are unusual events and represent approximately $1 \%$ of all oral malignant tumors. ${ }^{5}$ In most cases, metastatic lesions occur in the body of the mandible with a predilection for the posterior region (premolars and/or molars). ${ }^{5}$ The lungs (in men) and breast (in women) are the primary sites of carcinomas that metastasize to oral tissues. ${ }^{5}$ However, adrenal glands, kidneys, liver, prostate, the lower gastrointestinal tract, and the colorectal region are other common primary anatomic sites. ${ }^{5}$ Adenocarcinoma is the most common tumor of the colon that can affect the mandible (approximately $70 \%$ of cases), and in $7 \%$ of these metastatic cases, it may be the first clinical presentation of the disease. ${ }^{5}$ It is worth mentioning here that our patient had a history of oncologic treatment for this disease. In metastatic lesions, such as MAC, a destructive mandibular lesion detected in imaging examinations can be accompanied by other signs and symptoms (e.g., pain and soft tissue swelling), ${ }^{5}$ which were also found with the present case. The literature also includes paresthesia and tooth mobility in the main clinical signs and symptoms of metastatic carcinomas. ${ }^{5}$ Despite all the above considerations, we could not fail to consider the fact that our patient was an older woman and that her medical history included colon cancer. Thus, in our list of differential diagnosis, we considered MAC as a plausible candidate.

Intraoral sarcomas are very rare diseases and constitute approximately $1 \%$ of all head and neck tumors. ${ }^{6}$ Osteosarcoma is the most common malignant bone tumor, with extensive destructive potential. ${ }^{7}$ OSJs are aggressive mesenchymal tumors occurring in $6 \%$ to $8 \%$ of all cases of skeletal osteosarcomas. ${ }^{2}$ The disease usually affects adults in the third to fourth decades of life, with a male/female ratio of $1.4: 1 .^{2,7}$ In general, mandibular tumors normally occur in posterior region (molars and/or premolars). ${ }^{2}$ The main clinical finding of OSJ is the swelling of the affected bone and adjacent tissues., ${ }^{2,7}$ Because of the slowgrowing, painful mandibular swelling, which causes vestibular and lingual expansion of the mandibular body and angle, we considered the possibility of OSJ in our case. Radiographically, OSJ can manifest as radiolucent, radiopaque, or mixed (radiolucent-radiopaque) lesions. ${ }^{2,7}$ The classic ossification found growing in the adjacent soft tissue could show a "sunburst" appearance in some cases, but this is not considered a specific feature. ${ }^{7}$ In the radiographic examination of our patient, we observed a mixed bone lesion with a predominantly radiolucent aspect.

Another aggressive malignant bone tumor considered in our differential diagnosis was chondrosarcoma. This tumor has a low predilection for the head and neck region (1\%-3\%) and is found more commonly in the maxilla; the posterior mandible, ramus, nasal septum, and paranasal sinus are infrequent sites of occurrence. ${ }^{2}$ The disease incidence peaks in the seventh decade, but large variations exist in relation to age and time of diagnosis. $^{2}$ The predominant clinical signs and symptoms include a painless mass or a swelling with a long duration of pain. In the radiographic examination, these lesions are normally osteolytic and appear to be radiolucent, with ill-defined margins. ${ }^{2,8}$ Thus, we also considered chondrosarcoma in our differential diagnosis.

Osteoblastoma of the jaws, a rare benign bone tumor, occurs in about $85 \%$ of cases in patients under 30 years of age. ${ }^{2}$ A pathologic variant named "aggressive osteoblastoma" exhibits locally aggressive behavior, with a tendency to recur. ${ }^{9}$ In general, the cases appear in the posterior mandible and can show, radiographically, a radiolucent bone lesion with illdefined margins and irregular areas of mineralization. ${ }^{2}$

According to our clinical experience and those of several authors, ${ }^{3}$ our imaging findings, including the infiltrative and destructive bone lesions described here, had also been observed in malignant odontogenic lesions and other rare sarcomas, which was our preoperative diagnosis. Odontogenic carcinoma and sarcoma, as well as malignant fibrous histiocytoma, rhabdomyosarcoma, fibrosarcoma, liposarcoma, and angiosarcoma, are extremely rare conditions. ${ }^{10}$ Because of their rarity and tissue variety, estimating their anatomic predilection and clinical and pathologic features is not simple.,

\section{DIAGNOSIS AND MANAGEMENT}

An incisional biopsy of the lesion was performed under local anesthesia. Histopathologic examination revealed a malignant epithelial neoplasm with a low-grade follicular arrangement through dense connective tissue (Figure 3A). The neoplastic cells had a monotonous aspect, with round nuclei or with slightly irregular contour, with finely granular chromatin and evident nucleoli, peripherally displaced, without evidence of atypical mitotic figures and/or necrosis. The cytoplasm, which was present in moderate amount, varied from clear to slightly eosinophilic. In the lumens of follicles, there was discrete and dense eosinophilic proteinaceous material with a colloid-like aspect. There was no evidence of angiolymphatic or 

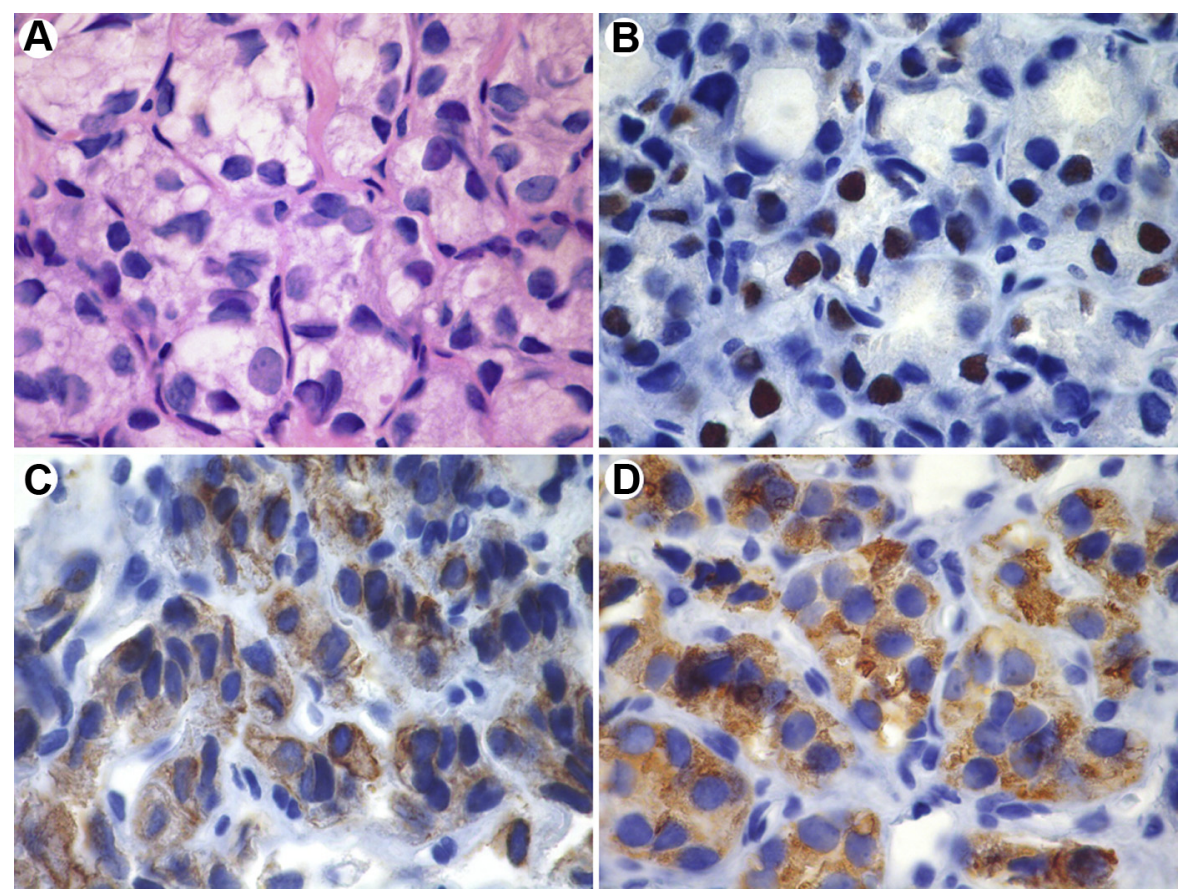

Fig. 3. Histopathologic features. A, The specimen shows a malignant neoplasm of epithelial origin, with a follicular architectural pattern. The follicles show columnar epithelial lining, and there is eosinophilic and amorphous material with colloid in the follicular lumens $(\mathrm{H} \& \mathrm{E}, \times 100)$. A high-resolution version of this slide for use with the Virtual Microscope is available as eSlide: VM00984. B, Immunohistochemistry reactions. Neoplastic epithelial cells show nuclear staining for TTF-1 $(\times 100)$. A high-resolution version of this slide for use with the Virtual Microscope is available as eSlide: VM00983. C, Analysis showing neoplastic epithelial cells with cytoplasmic positivity for CK-7 $(\times 100)$. A high-resolution version of this slide for use with the Virtual Microscope is available as eSlide: VM00981. D, Analysis showing cytoplasmic staining for thyroglobulin in neoplastic epithelial cells $(\times 100)$. A high-resolution version of this slide for use with the Virtual Microscope is available as eSlide: VM00982.

perineural invasion. Periodic acid-Schiff histochemical staining was performed, which more clearly showed the presence of this material in the follicular lumens, but not in the cytoplasm of neoplastic cells; thus, it was not a mucosecretory neoplasia. Morphologic findings favored metastatic neoplasia of epithelial origin, with the main diagnostic possibilities being follicular thyroid carcinoma, follicular variant of papillary thyroid carcinoma, and adenocarcinoma of unknown primary site, due to the follicular arrangement of the neoplasm. The possibility of its being a follicular variant of papillary thyroid carcinoma was less likely because this neoplasm often presents cells with nuclear slits, occasional intranuclear cytoplasmic pseudo-inclusions, and irregularity of nuclear contour. The morphology did not favor salivary gland neoplasia or metastasis of colorectal neoplasia. Because of the monotonous aspect and follicular/tubular arrangement of the neoplasia, it would be necessary to discard the diagnostic hypothesis of a neoplasm of neuroendocrine origin.

In an attempt to clarify the primary site of neoplasia, an immunohistochemical study was conducted, using the following panel of antibodies: TTF-1, thyroglobulin, CK7, CK20, Ki-67 (MIB-1), CEA, CDX2, chromogranin, and CD56. TTF-1 and thyroglobulin were performed to investigate a possible primary site in the thyroid; CK-7, CK20, CDX2, and CEA to investigate primary sites in the breast and gastrointestinal tract. The cellular proliferation index of neoplastic cells was assessed by using Ki-67 (MIB-1). Immunohistochemistry reactions showed positivity for TTF-1 (nuclear), CK-7 (cytoplasmic and membrane), and thyroglobulin (cytoplasmic) in the tumor cells (Figures 3B, 3C, and 3D). The cell proliferation index was less than 5\% (nuclear). The reactions for anti-CK20, CDX2, and CEA antibodies were negative, thus excluding the possibility of the primary site in the gastrointestinal tract. The reactions to chromogranin and CD56 were also negative, precluding the hypothesis of neoplasia of neuroendocrine origin. The positivity for thyroglobulin antibody excluded the possibility of a pulmonary primary site, even if TTF-1 and CK-7 were positive. Morphologic findings and positivity for TTF-1 and thyroglobulin did not favor the breast as the primary site. These results suggested metastasis from neoplasia of the thyroid gland. However, it was not possible to differentiate between the follicular variant of papillary carcinoma and follicular carcinoma. The hypothesis of 

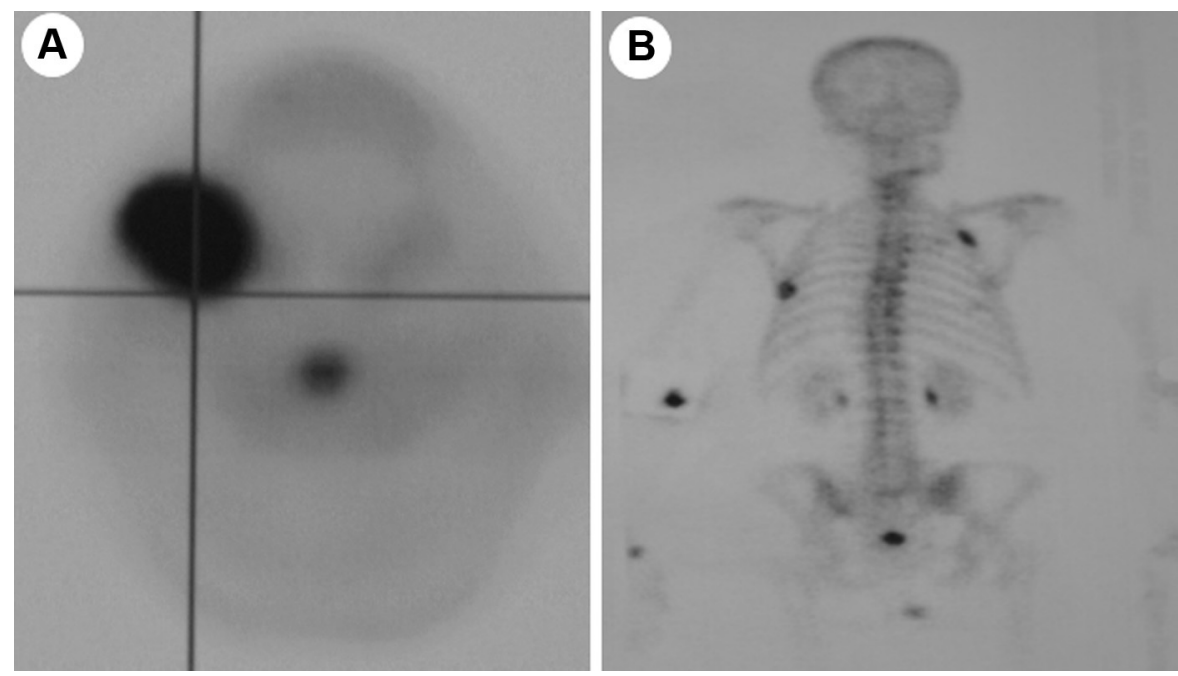

Fig. 4. Bone scintigraphy showing areas of increased uptake in the mandible on the right side (A) and ribs (B).

metastatic follicular thyroid carcinoma was considered because of the morphologic findings and positivity for TTF-1, CK-7, and thyroglobulin. Thus, the investigations for the primary tumor excluded lung, breast, and low gastrointestinal tract cancers, as well as recurrent colon cancer.

Bone scintigraphy (BS), using Tc99 m as radioisotope, showed areas of increased uptake in the body and angle of the mandible and ribs (Figure 4). Ultrasonography displayed a heterogeneous echographic texture due to the presence of nodes in the left thyroid lobe (calcified, $14 \mathrm{~mm}$ ) and in the right thyroid lobe (not calcified, $23 \mathrm{~mm}$ ) with regular contours. Scanning by positron emission tomography with 2-deoxy-2-[fluorine-18]fluoro-D-glucose with CT $\left({ }^{18}\right.$ F-FDG-PET-CT) showed glycolytic hypermetabolism in the mandibular lesion and bilateral ribs compatible with viable neoplastic tissue. The increased glycolytic metabolism in the node of the left thyroid lobe (shown by ${ }^{18} \mathrm{~F}$-FDG-PET-CT) made it worth performing a complementary examination using fineneedle aspiration. Fine-needle aspiration of the thyroid gland revealed a category IV follicular neoplasm. Hence, given the clinical, imaging, histopathologic, and immunohistochemical features, the final diagnosis of metastatic follicular thyroid carcinoma affecting the mandibular body and angle was made. The patient was then treated with total thyroidectomy complemented by radioiodine therapy and later with bisphosphonate treatment. Histopathologic examination of the surgical specimen confirmed the diagnosis of a follicular thyroid carcinoma (FTC). At the 1-year follow-up, a slight reduction of the metastatic lesion of the mandible was evident. So far, the patient has been in a relatively stable state of general health and is able to perform her activities of daily living.

\section{DISCUSSION}

In this report, we described the case of an older female patient with a destructive bone lesion in the mandible, which, to our surprise, was diagnosed as a metastatic FTC. Metastatic carcinomas are relatively uncommon, and the incidence of its occurrence in the maxillofacial region, especially in the mucosa and jaws, is low. ${ }^{5}$ According to Vural and Hanna, ${ }^{11}$ these neoplasms appear more frequently in the posterior body of the mandible (in the premolar and/or molar regions), ramus, and angle because of the increased vascularization in the region. The incidence of metastatic thyroid carcinoma in oral tissues is low (4\%-6.5\%), and nearly all recorded cases had occurred in the mandible. ${ }^{5}$ Nikitakis et al., ${ }^{12}$ in a review of 37 cases of metastatic thyroid carcinoma in oral tissues, observed a wide age range (13-87 years) among patients (mean age 60.6 years). In general, metastatic tumors were found to be located in the mandible $(86.5 \%)$, with a predilection for the ramus and angle; the majority of patients were females in their seventh decade of life, ${ }^{12}$ showing similar characteristics to our case. The authors attributed the female predilection to the higher incidence of thyroid cancer in women. Recently, Pingel et al. ${ }^{13}$ also showed that metastatic thyroid carcinoma in oral tissues was more common in women $(76 \%)$ and that most of the patients were between the sixth and eighth decades of life. In most cases, the jaws were involved (90\%), with a higher preference for the mandible $(82 \%){ }^{13}$

The histopathologic FTC variant is a welldifferentiated, low-grade malignant tumor, which originates in the follicular cells of the thyroid and resembles the microscopic pattern of the normal gland. ${ }^{11}$ It is the second most common cancer of the thyroid (after the 
papillary variant), occurring in $17 \%$ of all gland malignancies and is the most likely to spread. ${ }^{11}$ The study by Nikitakis et al. ${ }^{12}$ indicated that the histopathologic variant of thyroid carcinoma that most metastasized to oral tissues (55.5\% of cases) was the follicular type. Similarly, Pingel et al. ${ }^{13}$ found $54 \%$ of metastases to have originated from FTC. In addition, this tumor is associated with blood vessels, facilitating vascular invasion. Hematogenous dissemination is known to be the main source of distant metastases, with bones and lungs being the main targets (5\%-20\%). ${ }^{14}$ Biopsy of the mandibular lesion of our patient revealed a malignant epithelial neoplasm with a low-grade follicular arrangement. Immunopositivity for TTF-1, thyroglobulin, and CK-7 were also observed. The main immunohistochemical marker for FTC is thyroglobulin, which is present in over $95 \%$ of cases. ${ }^{15}$

In this report, the primary tumor was diagnosed only after identification of a metastatic lesion to the jaw. In about $23 \%$ to $33 \%$ of the time, a metastatic lesion to oral tissues was the first indication of a primary malignancy. ${ }^{5}$ However, Nikitakis et al., ${ }^{12}$ in analyzing the incidence rate of metastatic thyroid tumors to oral tissues, found a rate of over 50\% of metastases, which suggested that these tumors may be more likely to appear as the first sign of the disease, compared with other malignancies. These authors pointed out that in many cases, there was no history of previous tumors and/or growths in the neck or even previous thyroidectomy. However, it was also observed that some patients were diagnosed with thyroid cancer many years after detection of the metastatic lesion. ${ }^{12}$ It appears that these lesions can remain latent and unperceived for long periods of time. $^{12}$

The clinical signs and symptoms of our patient were swelling and pain. In general, metastatic tumors that occur in the mandible can also cause paresthesia, odynophagia, cervical lymphadenopathy, pathologic fractures, and tooth mobility. ${ }^{5}$ In addition, several authors have claimed that a peculiar site for the development of metastasis is the location and/or region of tooth extraction, with an observed a delay in wound repair. ${ }^{16}$ The less common clinical manifestations are mental nerve neuropathy (complaint of "numbness" in the chin), trismus, pain in the temporomandibular joint region, symptoms similar to trigeminal neuralgia, and mandibular osteomyelitis. ${ }^{5,16}$ A radiolucent lesion with irregular and ill-defined contours in the mandible was observed on the radiographic examination of our patient and 3-D reconstruction. The lesion presented expansive and destructive characteristics and was seen as an oval shape in the CT image. Approximately $5 \%$ of cases of metastases are undetectable by routine radiographic examination ${ }^{5}$; therefore most professionals resort to examination by BS. In the present case, BS detected lesions in the mandible and ribs, guiding the diagnosis of a metastatic tumor. Auxiliary imaging studies, such as angiography, arteriography, and Doppler ultrasonography, can contribute to detailing the rich blood supply present in the tumor. ${ }^{17}$ In our case, the ${ }^{18}$ F-FDG-PET-CT examination identified the glycolytic hypermetabolism, compatible with neoplastic tissue, in the mandibular lesion, ribs, and thyroid.

The differential diagnosis of metastatic tumors with irregular radiolucency may include various dental and/ or dentoalveolar inflammatory conditions, such as periapical lesions, severe periodontitis, periodontic-endodontic lesions, and pericoronitis. ${ }^{12}$ In the case of metastatic follicular carcinoma, arteriovenous malformations may be included because of the pulsation and/or auscultation caused by the rich vascularity usually present in the tumor. ${ }^{18}$

The treatment of metastatic thyroid carcinoma can be a combination of several therapeutic modalities, such as surgical resection, radioiodine therapy, radiotherapy, chemotherapy, and hormone therapy. ${ }^{12}$ Surgical treatment of the metastatic tumor is usually provided concomitantly with the removal of primary tumor (total thyroidectomy). ${ }^{11,12}$ Subsequently, radioiodine therapy and radiotherapy are implemented to enhance survival for the patient. ${ }^{12}$ However, the treatment decision rests on several variables, such as patient's suitability for surgery and/or the presence of multiple metastatic foci. ${ }^{11}$ In some cases, palliative treatment is the only feasible option. As noted earlier, our patient was treated with a total thyroidectomy and radioiodine therapy. Other authors have indicated that resection of a solitary bone metastasis in conjunction with total thyroidectomy increases the chances of patient survival, as a result of increased and more effective radioiodine absorption after surgery. ${ }^{14,16}$ However, it is noteworthy that surgical resection of secondary deposits is not always feasible.

The prognosis for patients with metastatic thyroid carcinoma is generally poor. In this context, an average of $40 \%$ of patients are alive 4 years after the diagnosis of the metastatic lesion, and $27 \%$ after 10 years in the case of tumors located in bone. ${ }^{19}$ Thus, bone metastasis represents a worse prognosis for thyroid carcinoma. . $^{13,20}$ Not many cases of this metastatic tumor to the mandible have been reported in the literature, and this is a limitation for precise determination of the prognosis. Thus, early detection of metastatic tumor and the primary FTC may increase the chances of overall survival and improve the results of treatment for each case.

Although it is a rare event, destructive mandibular lesions may represent the first manifestation of occult 
primary tumors of the thyroid gland. In general, this tumor is not included on the list of differential diagnoses of lesions in this bone region. A careful sequence of complementary examinations by a multidisciplinary team should be conducted for the diagnosis of thyroid metastatic lesion and its respective primary tumor.

The authors would like to thank to Dr. Mark W. Lingen, Department of Pathology, the University of Chicago, for his help with obtaining the histologic slides for the Virtual Microscope.

\section{REFERENCES}

1. Buchner A, Merrell PW, Carpenter WM. Relative frequency of central odontogenic tumors: a study of 1,088 cases from Northern California and comparison to studies from other parts of the world. J Oral Maxillofac Surg. 2006;64:1343-1352.

2. Neville BW, Damm DD, Allen CM, Bouquot JE. Oral and Maxillofacial Pathology. 3rd ed. St. Louis, MO: Saunders; 2009.

3. Adepitan AO, Bilodeau EA, Summersgill KF, Potluri A. Clinicopathologic review: multiple radiolucencies of the jaw bones. $\mathrm{Pa}$ Dent J (Harrisb). 2014;81:32-35.

4. Mankin HJ, Hornicek FJ, Ortiz-Cruz E, Villafuerte J, Gebhardt MC. Aneurysmal bone cyst: a review of 150 patients. J Clin Oncol. 2005;23:6756-6762.

5. Hirshberg A, Shnaiderman-Shapiro A, Kaplan I, Berger R. Metastatic tumours to the oral cavity - pathogenesis and analysis of 673 cases. Oral Oncol. 2008;44:743-752.

6. Gorsky M, Epstein JB. Head and neck and intra-oral soft tissue sarcomas. Oral Oncol. 1998;34:292-296.

7. Khorate MM, Goel S, Singh MP, Ahmed J. Osteosarcoma of mandible: a case report and review of literature. J Cancer Sci Ther. 2010;2:122-125.

8. Vencio EF, Reeve CM, Unni KK, Nascimento AG. Mesenchymal chondrosarcoma of the jaw bones: clinicopathologic study of 19 cases. Cancer. 1998;82:2350-2355.

9. Capodiferro S, Maiorano E, Giardina C, Lacaita MG, Lo Muzio L, Favia G. Osteoblastoma of the mandible: clinicopathologic study of four cases and literature review. Head Neck. 2005;27:616-621.

10. Sekerci AE, Nazlim S, Etoz M, Deniz K, Yasa Y. Odontogenic tumors: a collaborative study of 218 cases diagnosed over 12 years and comprehensive review of the literature. Med Oral Patol Oral Cir Bucal. 2015;20:E34-E44.

11. Vural E, Hanna E. Metastatic follicular thyroid carcinoma to the mandible: a case report and review of the literature. Am J Otolaryn. 1998;19:198-202.

12. Nikitakis NG, Polymeri A, Polymeris A, Sklavounou A. Metastatic papillary thyroid carcinoma to the maxilla: case report and literature review. Head Neck Pathol. 2012;6:216-223.

13. Pingel K, Kalogirou EM, Koutlas IG, Tosios KI. Mandibular metastasis of a papillary thyroid carcinoma, tall cell variant. case report and review of the literature. Hell Arch Oral Maxillofac Surg. 2013;3:143-152.

14. Grebe SK, Hay ID. Follicular thyroid cancer. Endocrinol Metab Clin North Am. 1995;24:761-801.

15. Tatic S. Histopathological and immunohistochemical features of thyroid carcinoma. Arch Oncol. 2003;11:173-174.

16. Pasupula AP, Dorankula SP, Thokala MR, Kumar MP. Metastatic follicular thyroid carcinoma to the mandible. Indian J Dent Res. 2012;23:843.

17. Araki M, Nishimura S, Iwanari S, et al. Mandibular metastases from follicular carcinoma of the thyroid gland: a case report. Oral Radiol. 2008;24:85-89.

18. Kumar RV, Chakravarthy C, Sekhar GR, Kare A. Metastatic thyroid carcinoma presenting as hypervascular lesion of the mandible: a case report and review of literature. J Oral Maxillofac Surg. 2010;68:2613-2616.

19. Ismail SB, Abraham MT, Zaini ZB, Yaacob HB, Zain RB. Metastatic follicular thyroid carcinoma to the mandible: a case report. Cases J. 2009;2:6533.

20. Brennan MD, Bergstralh EJ, van Heerden JA, McConahey WM. Follicular thyroid cancer treated at the Mayo Clinic, through 1970: initial manifestations, pathologic findings, therapy and outcome. Mayo Clin Proc. 1991;166:11-22.

\section{Reprint requests:}

Daniel Galera Bernabé, DDS, MS, PhD

Assistant Professor

Oral Oncology Center and Discipline of Stomatology

Department of Pathology and Clinical Propedeutics

Araçatuba Dental School

São Paulo State University - UNESP

1193 José Bonifácio St

Araçatuba

Sao Paulo 15050-015

Brazil

Danielbernabe@foa.unesp.br 